\title{
Convergence to equilibria in discrete population models
}

\author{
HASSAN A. EL-MORSHEDY $†$ and EDUARDO LIZ $\star^{*}$ \\ $\dagger$ Department of Mathematics, Damietta Faculty of Science, New Damietta 34517, Egypt \\ ‡Departamento de Matemática Aplicada II, E.T.S.I. Telecomunicación Campus Marcosende, \\ Universidad de Vigo, 36280 Vigo, Spain
}

(Received 5 April 2004; in final form 23 September 2004)

\begin{abstract}
For a family of difference equations $x_{n+1}=\alpha x_{n}+f\left(x_{n-k}\right), n=0,1, \ldots$, where $\alpha \in(0,1), k \in\{1,2, \ldots\}$, and $f:[0, \infty) \rightarrow(0, \infty)$ is continuous and decreasing, we find sufficient conditions for the convergence of all solutions to the unique positive equilibrium. In particular, we improve, up to our knowledge, all previous results on the global asymptotic stability of the equilibrium in the particular cases of the discrete MackeyGlass and Lasota-Wazewska models in blood-cells production.

Keywords: Difference equations; Global attractor; Discrete population models; Schwarzian derivative
\end{abstract}

Mathematics Subject Classificattion (2000): 39A10; 39A11; 92D25

\section{Introduction}

It is well-known that many discrete models in biology can be described by a difference equation

$$
x_{n+1}=\alpha x_{n}+f\left(x_{n-k}\right), \quad n=0,1, \ldots,
$$

where $\alpha \in(0,1), k \in\{1,2, \ldots\}$, and $f:[0, \infty) \rightarrow(0, \infty)$ is a continuous function. For some examples, see sections $4.5-4.7$ in the monograph [11]. Equation (1.1) is not only interesting by itself, but also as the discretization of an extensively studied family of delay differential equations, namely

$$
x^{\prime}(t)=-\delta x(t)+f(x(t-h)), \quad \delta>0, \quad h>0 .
$$

Relations between equations (1.1) and (1.2) can be found, for example, in refs. [1,10]. For processes modeled by equation (1.2), the reader can find many examples in the interesting list in [8, p. 78], including models in neurophysiology, metabolic regulation and agricultural commodity markets.

We will focus our attention on the case of a decreasing nonlinearity $f$. In fact, we will assume that $f$ is differentiable and $f^{\prime}(x)<0$ for all $x>0$. Under this hypothesis, it is clear that equation (1.1) has a unique constant solution $(\bar{x})$, where $\bar{x}$ solves the scalar equation

*Corresponding author. Email: eliz@dma.uvigo.es

Journal of Difference Equations and Applications

ISSN 1023-6198 print/ISSN 1563-5120 online (C) 2005 Taylor \& Francis Ltd

http://www.tandf.co.uk/journals

DOI: $10.1080 / 10236190512331319334$ 
$h(x)=x$, with $h(x)=(1-\alpha)^{-1} f(x)$. For any initial string $\left\{x_{-k}, \ldots, x_{0}\right\}$ with $x_{i} \geq 0, i=$ $-k, \ldots, 0$, the solution of equation (1.1) is a sequence $\left\{x_{n}\right\}_{n \geq-k}$ constructed by induction, and it is positive. We will only consider these solutions. Our aim in this paper consists in finding sufficient conditions to ensure that all solutions of equation (1.1) converge to $\bar{x}$. In this case, we will say that $\bar{x}$ is a global attractor of equation (1.1).

Our research is mainly motivated by two well-known examples of equation (1.1) with decreasing $f$, namely, the discrete analogue of the Mackey-Glass equation in haematopoiesis

$$
x_{n+1}=\alpha x_{n}+\frac{\beta}{1+x_{n-k}^{p}}, \quad n=0,1, \ldots,
$$

where $\alpha \in(0,1)$ and $p, \beta$ are positive constants, and the discrete analogue of the LasotaWazewska model for the survival of red blood cells in an animal

$$
x_{n+1}=\alpha x_{n}+p e^{-q x_{n-k}}, \quad n=0,1, \ldots,
$$

with $p, q>0$. For these equations, sufficient conditions to ensure the convergence of all positive solutions to the equilibrium were obtained in recent years [2,5-7,9-12]. By rigorous comparisons with these papers, we will show that our results improve all conditions stated there for the particular cases of equations (1.3) and (1.4).

A well-known result [11, Corollary 2.4.1] establishes that, for decreasing $f$, all positive solutions of equation (1.1) converge to the unique equilibrium $\bar{x}$ if $\bar{x}$ is a global attractor for the discrete dynamical system generated by $h$, that is, if the sequence defined by $x_{n+1}=$ $h\left(x_{n}\right), n \geq 0$, converges to $\bar{x}$ for every $x_{0} \geq 0$. (In fact, this result has a more general form in ref. [9, Theorem 1], where it is only required the existence of an invariant closed interval for $h$ containing the attracting fixed point $\bar{x}$ ).

It can be checked $[4,6,9]$ that the nonlinearities in equations (1.3) and (1.4) not only are decreasing but also they have negative Schwarzian derivative. This property makes the study of the global stability in equations (1.3) and (1.4) easier. The following hypothesis will be required when necessary:

(H) $f \in C^{3}([0, \infty),(0, \infty)), f^{\prime}(x)<0,(S f)(x)<0$ for all $x>0$, where $(S f)(x)$ is the Schwarzian derivative of $f$ at $x$, defined by

$$
(S f)(x)=\frac{f^{\prime \prime \prime}(x)}{f^{\prime}(x)}-\frac{3}{2}\left(\frac{f^{\prime \prime}(x)}{f^{\prime}(x)}\right)^{2} .
$$

Denote $c:=\left|h^{\prime}(\bar{x})\right|$. As it was pointed out in ref. [2] (see also refs. [6,9]), under hypothesis $(\mathrm{H}), \bar{x}$ is a global attractor for $h$ if and only if $c \leq 1$. Hence, the following result holds:

Proposition $1.1[2,6]$ If $c \leq 1$ and $(H)$ holds, then $\bar{x}$ is a global attractor of equation (1.1).

Let us observe that Proposition 1.1 provides a condition independent of the size of the delay $k$ (depends only on $\alpha$ and $f$ ). Moreover, condition $c \leq 1$ is the sharpest condition for the global asymptotic stability independent of $k$ (often called absolute stability). Indeed, the linearized equation of (1.1) at $\bar{x}$ is

$$
x_{n+1}=\alpha x_{n}+f^{\prime}(\bar{x}) x_{n-k}, \quad n=0,1, \ldots,
$$


According to ref. [14, Theorem 4], this equation is asymptotically stable if and only if

$$
\left|f^{\prime}(\bar{x})\right|<\left(\alpha^{2}+1-2 \alpha \cos \theta\right)^{1 / 2},
$$

where $\theta$ is the solution in $(0, \pi / k+1))$ of $\sin (k \theta) / \sin [(k+1) \theta]=1 / \alpha$. Hence, it is easily seen that if $c>1$, there exists an integer $k>0$ such that inequality (1.6) is violated (and therefore $\bar{x}$ is unstable).

Since Proposition 1.1 gives a criterion for the absolute stability in equation (1.1), we will focus our attention in obtaining delay-dependent conditions for the global attractivity of the equilibrium.

In recent years, several authors made relevant contributions in this direction. In [12], Krause and Pituk have obtained a global stability result for equation (1.1) (without assuming that $f$ is decreasing), by using an elegant approach based on monotonicity arguments. Theorem 7 in ref. [12] ensures the convergence of all solutions to the equilibrium under condition

$$
\max _{x>0}\left\{\left|f^{\prime}(x)\right|\right\} \leq \alpha^{k+1} \frac{k^{k}}{(k+1)^{(k+1)}} .
$$

Our theorem 2.7 below proves the convergence to $\bar{x}$ of all solutions of equation (1.1) under condition

$$
\max _{x \in[a, b]}\left\{\left|f^{\prime}(x)\right|\right\} \leq \alpha^{k+1} \frac{3 k+4}{2(k+1)^{2}},
$$

where $a, b$ satisfy $0<a<\bar{x}<b$, and will be defined in the second section. Since equation (1.8) is sharper than equation (1.7), for $f$ decreasing our Theorem 2.7 improves ref. [12, Theorem 7].

Using Liapunov functions, Graef and Qian proved in [5] the convergence of all solutions to the equilibrium $\bar{x}$ of equation (1.1) with decreasing $f$ under the following condition (also sharper than equation (1.7)):

$$
\max _{x \geq 0}\left\{\left|f^{\prime}(x)\right|\right\} \leq \frac{1-\alpha}{1-\alpha^{k+1}} .
$$

Let us observe that equations (1.7)-(1.9) require the estimation of $f^{\prime}(x)$ at least on a real interval containing $\bar{x}$, while Proposition 1.1 only involves the value of $f^{\prime}$ at the equilibrium point $\bar{x}$. The consideration of hypothesis $(\mathrm{H})$ permits to obtain results in this direction, as shown in Gyôri and Trofimchuk [6]. The following statement is equivalent to their Corollary 17:

THEOREm $1.2[6]$ Assume that $c>1$ and $(\mathrm{H})$ holds. Then $\bar{x}$ is a global attractor of equation (1.1) if

$$
\alpha^{k+1}>\frac{c^{2}-c}{c^{2}+1} .
$$

In fact, the statement in [6] is more general, since it applies also to unimodal nonlinearities with negative Schwarzian. Next, it is a matter of calculations to check that Theorem 1.2 improves the results in [5] for $f$ satisfying $(\mathrm{H})$, and, in particular, it gives better results for equations (1.3) and (1.4). Indeed, equation (1.9) requires that

$$
\left|f^{\prime}(\bar{x})\right| \leq \frac{1-\alpha}{1-\alpha^{k+1}},
$$


which, for $c \geq 1$, is equivalent to

$$
\alpha^{k+1} \geq \frac{c-1}{c} .
$$

This condition is stronger than inequality (1.10).

Recently, in ref. [2] a new global stability result was proved which applies in some cases for which equation (1.10) does not hold. However, both results are not comparable. Our main result in this paper extends Theorem 3.1 in ref. [2] and, in particular, improves Theorem 1.2 when $f$ satisfies $(\mathrm{H})$. As a result, we find sharper conditions for the convergence of all positive solutions of equations (1.3) and (1.4) to the corresponding equilibria. Some of our conditions involve the estimation of $f^{\prime}(x)$ on a certain compact interval $[a, b] \subset$ $(0, \infty)$.

The paper is organized as follows: in section 2, we state and prove some results on the global attraction of $\bar{x}$ without assuming condition (H). Next, in section 3, we apply the results from the second section to the particular situation when $(\mathrm{H})$ holds, improving, up to our knowledge, all previous results for this class of difference equations. Moreover, we establish some comparisons among our estimations and the conditions for the local asymptotic stability of $\bar{x}$, which lead us to formulate some conjectures and open problems. Finally, in section 4, we apply our main result to equations (1.3) and (1.4).

\section{Main results for $f$ decreasing}

Throughout this section, we do not assume that condition (H) holds, but only that $f^{\prime}$ is negative. Since $h$ is decreasing, $\bar{x}$ is a global attractor of equation (1.1) under the assumption that $\bar{x}$ is the unique fixed point of $h^{2}=h \circ h$. Hence, we can assume that $h^{2}$ has more than one fixed point. In this case, we can define the numbers

$$
a=\inf \left\{x>0: h^{2}(x)=x\right\}, \quad b=\sup \left\{x>0: h^{2}(x)=x\right\} .
$$

Moreover [2], for a given solution $\left\{x_{n}\right\}$ of equation (1.1),

$$
a \leq \liminf _{n \rightarrow \infty} x_{n}=L \leq S=\limsup _{n \rightarrow \infty} x_{n} \leq b .
$$

Next, by induction one can choose integer subsequences $\left\{n_{l}\right\}$ and $\left\{n_{l}^{\prime}\right\}$, with $\lim _{l \rightarrow \infty}$ $n_{l}=\lim _{l \rightarrow \infty} n_{l}^{\prime}=\infty$, such that

$$
\lim _{l \rightarrow \infty} x_{n_{l}+1}=L, \lim _{l \rightarrow \infty} x_{n_{l}-i}=L_{i}, \lim _{l \rightarrow \infty} x_{n_{l}^{\prime}+1}=S, \quad \lim _{l \rightarrow \infty} x_{n_{l}^{\prime}-i}=S_{i},
$$

with $L_{i}, S_{i} \in[L, S]$ for all $i=0,1,2, \ldots, 2 k$.

Since any solution of equation (1.1) is also a solution of the higher order equation [2, p. 754],

$$
x_{n+1}=F\left(x_{n-k}\right)+(1-\alpha) \sum_{i=1}^{k} \alpha^{i} h\left(x_{n-k-i}\right), \quad n \geq 2 k,
$$


where $F(x)=\alpha^{k+1} x+(1-\alpha) h(x)$ for all $x \in[a, b]$. Then, taking the limits in both sides of equations (1.1) and (2.1) through $n_{l}$ and $n_{l}^{\prime}$, we obtain the following equations:

$$
\begin{gathered}
L=\alpha L_{0}+(1-\alpha) h\left(L_{k}\right), \\
S=\alpha S_{0}+(1-\alpha) h\left(S_{k}\right), \\
L=F\left(L_{k}\right)+(1-\alpha) \sum_{i=1}^{k} \alpha^{i} h\left(L_{k+i}\right),
\end{gathered}
$$

and

$$
S=F\left(S_{k}\right)+(1-\alpha) \sum_{i=1}^{k} \alpha^{i} h\left(S_{k+i}\right) .
$$

Since $h(x)=x$ only if $x=\bar{x}$, then, assuming $L<S, F$ must satisfy the relations $\left(1-\alpha+\alpha^{k+1}\right) h(S)<F(x)<\left(1-\alpha+\alpha^{k+1}\right) h(L) \quad$ for $\quad$ all $\quad x \in[L, S] . \quad$ Therefore, equations (2.4) and (2.5) yield

$$
L>h(S) \text { and } S<h(L)
$$

and hence there exist two numbers $c_{1} \in[L, \bar{x}), c_{2} \in(\bar{x}, S]$ such that $h\left(c_{1}\right)=S$ and $h\left(c_{2}\right)=L$. Also, equation (2.6) implies that $L>h^{2}(L)$ and $S<h^{2}(S)$; that is $L, S \in(a, b)$.

Lemma 2.1 Assume that the numbers $c_{1}, c_{2}, L, L_{i}, S, S_{i}$ are defined as before such that $L<S$. Then $L_{k} \in\left[c_{2}, S\right]$ and $S_{k} \in\left[L, c_{1}\right]$.

Proof Since $L_{0} \geq L$ and $S_{0} \leq S$, then equations (2.2) and (2.3) yield

$$
L \geq h\left(L_{k}\right) \quad \text { and } \quad S \leq h\left(S_{k}\right)
$$

or equivalently,

$$
h\left(c_{2}\right) \geq h\left(L_{k}\right) \quad \text { and } \quad h\left(c_{1}\right) \leq h\left(S_{k}\right) .
$$

Our assertion follows now from the decreasing nature of $h$.

Let us now define the family of functions $F_{\lambda}$ on $[a, b]$ as follows:

$$
F_{\lambda}(x)=\lambda \alpha^{k+1} x+(1-\alpha) h(x)=F(x)-(1-\lambda) \alpha^{k+1} x, \quad \lambda \geq 0 .
$$

Then

$$
F_{\lambda}^{\prime}(x)=\lambda \alpha^{k+1}+(1-\alpha) h^{\prime}(x) .
$$

For convenience in the presentation of our next results, we state the following lemma, whose proof is an easy consequence of the previous relation.

LEMMA 2.2 The following statements hold:

(a) $F_{\lambda}^{\prime}(x) \leq 0$ for all $x \in[a, b]$ if and only if

$$
\min _{x \in[a, b]}\left\{\left|f^{\prime}(x)\right|\right\} \geq \lambda \alpha^{k+1} .
$$


(b) $F_{\lambda}^{\prime}(x) \geq 0$ for all $x \in[a, b]$ if and only if

$$
\max _{x \in[a, b]}\left\{\left|f^{\prime}(x)\right|\right\} \leq \lambda \alpha^{k+1} .
$$

Next, equations (2.4) and (2.5) can be rewritten as follows:

$$
L=F_{\lambda}\left(L_{k}\right)+(1-\lambda) \alpha^{k+1} L_{k}+(1-\alpha) \sum_{i=1}^{k} \alpha^{i} h\left(L_{k+i}\right),
$$

and

$$
S=F_{\lambda}\left(S_{k}\right)+(1-\lambda) \alpha^{k+1} S_{k}+(1-\alpha) \sum_{i=1}^{k} \alpha^{i} h\left(S_{k+i}\right)
$$

We are now ready to state our main results.

Theorem 2.3 Let $\lambda \in[0,1]$ be such that equation (2.7) holds. Define $G(x)=$ $\lambda \alpha^{k+1} x+\left(1-\alpha^{k+1}\right) h(x), \Psi(x)=x-(1-\lambda) \alpha^{k+1} h^{-1}(x)$, and $V(x)=\Psi^{-1}(G(x))$ for all $x \in[a, b]$ where $h^{-1}$ is the inverse of $h$. If $\bar{x}$ is the unique fixed point of $V^{2}$ in $(a, b)$, then $\bar{x}$ is a global attractor of all solutions of equation (1.1)

Proof Assume that $\left\{x_{n}\right\}$ is any solution of equation (1.1) such that $L<S$. From Lemma $2.2, F_{\lambda}$ is nonincreasing on $[a, b]$. Hence equations (2.9), (2.10) and Lemma 2.1 yield

$$
L \geq F_{\lambda}(S)+(1-\lambda) \alpha^{k+1} L_{k}+\left(\alpha-\alpha^{k+1}\right) h(S) \geq(1-\lambda) \alpha^{k+1} h^{-1}(L)+G(S),
$$

and

$$
S \leq F_{\lambda}(L)+(1-\lambda) \alpha^{k+1} S_{k}+\left(\alpha-\alpha^{k+1}\right) h(L) \leq(1-\lambda) \alpha^{k+1} h^{-1}(S)+G(L) .
$$

Rearranging, and taking into account that $\Psi$ is increasing, we obtain $L \geq V(S)$ and $S \leq V(L)$, and hence, since $V$ is decreasing,

$$
L \geq V^{2}(L) \quad \text { and } \quad S \leq V^{2}(S),
$$

which, in view of the fact that $V^{2}([a, B]) \subseteq(a, b)$, lead to the existence of a fixed point of the map $V^{2}$ in $(a, b)$ different from $\bar{x}$. This contradiction proves that $L=S=\bar{x}$, and hence $\lim _{n \rightarrow \infty} x_{n}=\bar{x}$ for all solution $\left\{x_{n}\right\}$ of equation (1.1).

We notice that, for $\lambda=1$, Theorem 2.3 gives Theorem 3.1 in [2].

Theorem 2.4 Let $\lambda \geq 1$ be such that equation (2.8) holds. Define $Z(x)=(1-\lambda) \alpha^{k} x+$ $\left(1-\alpha^{k}\right) h(x), \Phi(x)=x-\lambda \alpha^{k} h^{-1}(x)$, and $H(x)=\Phi^{-1}(Z(x))$ for all $x \in[a, b]$, where $h^{-1}$ is the inverse of $h$. If $\bar{x}$ is the unique fixed point of $H^{2}$ in $(a, b)$, then $\bar{x}$ is a global attractor of all solutions of equation (1.1).

Proof Proceeding as in the proof of the above theorem, the increasing nature of $F_{\lambda}$ on $[a, b]$, equation (2.9) and Lemma 1 yield

$$
L \geq F_{\lambda}\left(c_{2}\right)+\alpha Z(S)=\lambda \alpha^{k+1} h^{-1}(L)+(1-\alpha) L+\alpha Z(S) .
$$

Rearranging, we obtain $L-\lambda \alpha^{k} h^{-1}(L) \geq Z(S)$, and hence, since $\Phi$ is increasing,

$$
L \geq \Phi^{-1}(Z(S))=H(S) \text {. }
$$


Using similar arguments with equation (2.10), it follows that $S \leq H(L)$. Therefore,

$$
L \geq H^{2}(L) \text { and } S \leq H^{2}(S) .
$$

Since $H^{2}([a, b]) \subseteq(a, b)$, it follows that $H^{2}$ has a fixed point in $(a, b)$ different from $\bar{x}$, which contradicts our assumptions. The proof is complete.

Next we assume that $F_{\lambda}$ has only one critical point in $[a, b]$, say $x_{\lambda}^{*}$, which will be a local minimum.

TheOREm 2.5 Assume that $\lambda \in[0,1], x_{\lambda}^{*}, V$ are defined as before,

$$
F_{\lambda}^{\prime}(\bar{x}) \leq 0 \quad \text { and either } \quad V\left(F_{\lambda}\left(x_{\lambda}^{*}\right)\right) \leq x_{\lambda}^{*} \quad \text { or } \quad V(a) \leq x_{\lambda}^{*} .
$$

If the map $V^{2}$ has no fixed points in $(a, b)$ different from $\bar{x}$, then $\bar{x}$ is a global attractor of all solutions of equation (1.1).

Proof Let us assume that $\left\{x_{n}\right\}$ be any solution of equation (1.1) such that $L<S$. Since $F_{\lambda}^{\prime}(\bar{x}) \leq 0$, then $\bar{x} \leq x_{\lambda}^{*}$. Thus, $F_{\lambda}(x)$ is decreasing on $[L, \bar{x}]$, and equation (2.10) yields $S \leq V(L)$. Next, since $L>a$ and $L>F_{\lambda}\left(x_{\lambda}^{*}\right)$ (from equation (2.9)), it follows from equation (2.11) that either $S<V\left(F_{\lambda}\left(x_{\lambda}^{*}\right)\right) \leq x_{\lambda}^{*}$ or $S<V(a) \leq x_{\lambda}^{*}$. This means that $F_{\lambda}$ is decreasing on $[L, S]$. Therefore, the proof can be completed as the proof of Theorem 2.3.

THEOREM 2.6 Assume that $x_{\lambda}^{*}$ and $H$ are defined as before. If

$$
F_{1}\left(x_{1}^{*}\right)+\left(\alpha-\alpha^{k+1}\right) a \geq x_{1}^{*}
$$

and, for $\lambda=1$, the map $H^{2}$ has no fixed points in $(a, b)$ different from $\bar{x}$, then $\bar{x}$ is a global attractor of all solutions of equation (1.1).

Proof As usual, we assume that $L<S$. Then equations (2.9) and (2.12) yield

$$
L \geq F_{1}\left(x_{1}^{*}\right)+\left(\alpha-\alpha^{k+1}\right) a \geq x_{1}^{*} .
$$

Therefore, $F_{1}^{\prime}(x) \geq 0$ for all $x \in[L, S]$, and hence the proof can be completed using the same reasoning as in the proof of Theorem 2.4, but with $\lambda=1$.

In addition to the above technique, one may also apply linear methods to obtain global attractivity criteria for equation (1.1). First, we have to prove that any solution of equation (1.1), say $\left\{x_{n}\right\}$, is a solution of a corresponding linear equation. This can be done using the mean value theorem, which implies $f\left(x_{n-k}\right)=f(\bar{x})+\left(x_{n-k}-\bar{x}\right) f^{\prime}\left(\xi_{n}\right)$, where $\xi_{n}$ lies between $\bar{x}$ and $x_{n-k}$. Therefore, from equation (1.1), we obtain

$$
x_{n+1}-\alpha x_{n}=(1-\alpha) \bar{x}+\left(x_{n-k}-\bar{x}\right) f^{\prime}\left(\xi_{n}\right) .
$$

With this idea, we can prove the following result:

THeORem 2.7 Assume that

$$
\max _{x \in[a, b]}\left\{\left|f^{\prime}(x)\right|\right\} \leq \alpha^{k+1} \frac{3 k+4}{2(k+1)^{2}} .
$$

Then the equilibrium point $\bar{x}$ is a global attractor of all solutions of equation (1.1). 
Proof According the remark stated in ref. [3, p. 160], if $\left\{p_{n}\right\}$ is a sequence of nonnegative real numbers and the inequality

$$
\sum_{i=n-k}^{n} p_{i} \leq \frac{3}{2}+\frac{1}{2(k+1)}
$$

eventually holds, then every solution of the difference equation

$$
x_{n+1}-x_{n}+p_{n} x_{n-k}=0, \quad n=0,1, \ldots
$$

is bounded.

Let $\left\{x_{n}\right\}$ be a given solution of equation (1.1). Then, using the above reasoning, we get that $\left\{z_{n}\right\}=\left\{\left(x_{n}-\bar{x}\right) \alpha^{-n}\right\}$ satisfies

$$
z_{n+1}-z_{n}+\alpha^{-k-1}\left|f^{\prime}\left(\xi_{n}\right)\right| z_{n-k}=0,
$$

for some $\xi_{n}$ between $\bar{x}$ and $x_{n-k}$. As $L, S \in(a, b)$, then a positive integer $n_{0}$ exists such that $x_{n-k} \in(a, b)$ for all $n \geq n_{0}$. Thus $\xi_{n} \in(a, b)$ for all $n \geq n_{0}$, and hence inequality (2.13) implies that the sequence $\left\{p_{n}\right\}$ defined by $p_{n}=\alpha^{-k-1}\left|f^{\prime}\left(\xi_{n}\right)\right|$ satisfies equation (2.14) for $n$ large. Hence, $\left\{z_{n}\right\}$ is bounded, and therefore, since $\alpha \in(0,1),\left\{x_{n}\right\}$ converges to $\bar{x}$.

\section{The case of negative Schwarzian}

In this section, we assume that hypothesis $(\mathrm{H})$ holds. In this case, one can state easier and more explicit global attractivity criteria from the above general results. We need two previous lemmas. The first of them shows that, when $(\mathrm{H})$ holds, the function $h$ can have only one 2-cycle.

Lemma 3.1 Assume that $g:[0, \infty) \rightarrow(0, \infty)$ satisfies $(S g)(x)<0$ and $g^{\prime}(x)<0$ for all $x>0$, and let $\bar{x}$ be the unique solution of $g(x)=x$. If $\left|g^{\prime}(\bar{x})\right|>1$, then there exists a unique pair $(a, b) \in(0, \infty)^{2}, a<\bar{x}<b$, such that $g(a)=b$ and $g(b)=a$.

Proof Existence of $(a, b)$ follows from ref. [2, Lemma 3.3]; uniqueness is a straightforward consequence of Lemma 2.6 in ref. [16].

Lemma 3.2 Let $I=[a, b]$ be a real interval, and assume that $g: I \rightarrow I$ satisfies $(S g)(x)<0$ for all $x \in I$. Let $g_{\lambda}(x)=\lambda x+g(x)$ with $\lambda>0$. If $g_{\lambda}^{\prime}(x)<0$ for all $x \in I$, then $\left(S g_{\lambda}\right)(x)<0$ for all $x \in I$.

Proof First we notice that $g_{\lambda}^{\prime}(x)<0$ for all $x \in I$ implies that $g^{\prime}(x)<0$ for all $x \in I$. Since $g_{\lambda}^{\prime}(x)<0,\left(S g_{\lambda}\right)(x)<0$ if and only if $g_{\lambda}^{\prime}(x) g_{\lambda}^{\prime \prime \prime}(x)<(3 / 2)\left(g_{\lambda}^{\prime \prime}(x)\right)^{2}$. In particular, $\left(S g_{\lambda}\right)(x)<0$ if $g_{\lambda}^{\prime \prime \prime}(x)=g^{\prime \prime \prime}(x)>0$. Hence, we can assume that $g^{\prime \prime \prime}(x) \leq 0$. Since $g_{\lambda}^{\prime}(x)=\lambda+g^{\prime}(x)$, $g_{\lambda}^{\prime \prime}(x)=g^{\prime \prime}(x)$, and $g_{\lambda}^{\prime \prime \prime}(x)=g^{\prime \prime \prime}(x)$, it follows that $g_{\lambda}^{\prime}(x) g_{\lambda}^{\prime \prime \prime}(x) \leq g^{\prime}(x) g^{\prime \prime \prime}(x)<(3 / 2) \times$ $\left(g^{\prime \prime}(x)\right)^{2}=(3 / 2)\left(g_{\lambda}^{\prime \prime}(x)\right)^{2}$, and therefore $\left(S g_{\lambda}\right)(x)<0$ for all $x \in I$.

Lemma 3.2 ensures that $(S G)(x)<0$ for all $x \in[a, b]$ provided that $F_{\lambda}^{\prime}(x) \leq 0$ for all $x \in[a, b]$, that is, when equation (2.7) holds. Moreover, as in ref. [6, Section 3.1], one can see that $\left(S \Psi^{-1}\right)(x)<0,\left(S \Phi^{-1}\right)(x)<0$ for all $x>0$. Consequently, the known formula

$$
(S(f \circ g))(x)=S(f)(g(x))\left(g^{\prime}(x)\right)^{2}+(S g)(x)
$$


implies that $(S V)(x)<0$ for all $x \in[a, b]$ when equation (2.7) holds, and that $(S H)(x)<0$ for all $x \in[a, b]$ as long as $(S Z)(x) \leq 0$ for all $x \in[a, b]$.

On the other hand, since $f$ is decreasing and has negative Schwarzian, Proposition 2.4 in ref. [16] ensures that $f^{\prime}$ cannot have a local maximum. Therefore, $\min \left\{\left|f^{\prime}(x)\right|: x \in\right.$ $[a, b]\}=\min \left\{\left|f^{\prime}(a)\right|,\left|f^{\prime}(b)\right|\right\}$. Hence, equation (2.7) reads

$$
\min \left\{\left|f^{\prime}(a)\right|,\left|f^{\prime}(b)\right|\right\} \geq \lambda \alpha^{k+1},
$$

where $\{a, b\}$ is the unique 2-cycle of $h=(1-\alpha)^{-1} f$.

Now, we can prove the following result:

THEOREM 3.3 Assume that $(H)$ holds. Then all solutions of equation (1.1) converge to the equilibrium point $\bar{x}$ if either $c \leq 1$ or $c>1$ and one of the following conditions is satisfied:

(1) There exists $\lambda \in[0,1]$ such that equation (3.1) holds and

$$
\alpha^{k+1} \geq \frac{c^{2}-c}{\lambda(c-1)+c^{2}+1} .
$$

(2) There exists $\lambda \geq 1$ such that equation (2.8) holds, (SZ)(x) $\leq 0$ for all $x \in[a, b]$, and

$$
\alpha^{k} \geq \frac{c^{2}-c}{\lambda(1-c)+c^{2}+c} .
$$

(3) $G^{\prime}(x)<0$ for all $x \in[a, b]$ and equations (2.11), (3.2) hold for some $\lambda \in[0,1]$.

(4) Condition (2.12) holds and

$$
\alpha^{k} \geq \frac{c^{2}-c}{c^{2}+1}
$$

Proof Let $\varphi:[a, b] \rightarrow[a, b]$ be a $C^{3}$ map such that $\varphi^{\prime}(x)<0,(S \varphi)(x)<0$ for all $x \in$ $(a, b)$, and let $\bar{x}$ be the unique fixed point of $\varphi$ in $(a, b)$. According to ref. [15, Proposition 3.3] (see also ref. [2, Lemma 3.2]), $\bar{x}$ is the unique fixed point of $\varphi^{2}$ in $[a, b]$ if and only if $\varphi^{\prime}(\bar{x}) \geq-1$.

To prove part (1), let $V$ be the function defined in the statement of Theorem 2.3. It is clear that $V^{\prime}(x)<0$ for all $x \in(a, b)$. Moreover, by the above reasonings, $(S V)(x)<0$ for all $x \in[a, b]$ when equation (3.1) holds. Hence, in view of Theorem 2.3, we only have to show that $V^{\prime}(x) \geq-1$ if equation (3.2) is satisfied. Indeed, since $V(x)=\Psi^{-1}(G(x))$, it follows that $G^{\prime}(x)=\Psi^{\prime}(V(x)) V^{\prime}(x)$ for all $x \in(a, b)$; in particular, $V^{\prime}(\bar{x})=G^{\prime}(\bar{x}) /$ $\Psi^{\prime}(\bar{x}) \geq-1$ if and only if $G^{\prime}(\bar{x}) \geq-\Psi^{\prime}(\bar{x})$. It is easy to check that this inequality is equivalent to equation (3.2).

The proofs of items (2)-(4) follow in an analogous way, by using Theorems 2.4-2.6, respectively.

Remark 3.4 Since $\alpha^{-k-1} \min \left\{\left|f^{\prime}(a)\right|,\left|f^{\prime}(b)\right|\right\}>0$, we can always take a positive $\lambda$ in equation (3.2) for which equation (3.1) holds. Hence, Theorem 3.3 improves Theorem 1.2.

The sharpest form of equation (3.2) is reached for $\lambda=1$, and it takes the form

$$
\alpha^{k+1} \geq \frac{c-1}{c+1} .
$$


Thus, we have the following consequence of Theorem 3.3 (1):

COROllary 3.5 Assume that $c>1,(H)$ holds, and

$$
\alpha^{k+1} \leq \min \left\{\left|f^{\prime}(a)\right|,\left|f^{\prime}(b)\right|\right\}
$$

Then equation (3.5) implies that $\bar{x}$ is a global attractor for equation (1.1).

On the other hand, since $(S Z)(x)<0$ for all $x>0$ when $\lambda=1$, condition (3.4) is sufficient for the global attractivity if equation (2.8) holds for $\lambda=1$. Hence, we have the following consequence from Theorem 3.3 (2):

COROLlary 3.6 Assume that $c>1,(H)$ holds, and

$$
\alpha^{k+1} \geq \max _{x \in[a, b]}\left\{\left|f^{\prime}(x)\right|\right\}
$$

Then equation (3.4) implies that $\bar{x}$ is a global attractor for equation (1.1).

Notice that checking conditions (3.6) and (3.7) require to estimate the 2-cycle of $h$. However, this is not a difficult task for particular cases of equation (1.1) using computer programs (see the numerical examples in section 4).

Remark 3.7 Elementary calculations show that Corollary 3.6 gives better results than Theorem 2.7 whenever $k=1$, or $k>1$ and $\alpha \leq \alpha_{1}$, where $\alpha_{1}$ is the unique root in $(0,1)$ of equation $\left(4-4 p-p^{2}\right) x^{2}+4(p-2) x+4=0$, with $p=p(k)=(3 k+4) /\left(2(k+1)^{2}\right)$. For $\alpha$ close to 1 , it seems that both results are not comparable, so Theorem 2.7 is of independent interest even for $f$ satisfying (H).

On the other hand, condition (3.7) implies equation (3.4) whenever

$$
\alpha \leq \alpha_{0}:=2(\sqrt{2}-1)=0.828427 \ldots
$$

Hence, we have the following consequence of Corollary 3.6:

COROLlary 3.8 Assume that $c>1,(H)$ holds, and $\alpha \leq \alpha_{0}$. Then condition (3.7) implies that $\bar{x}$ is a global attractor for equation (1.1).

It is interesting to compare our global attractivity conditions with the criterion for the local asymptotic stability of $\bar{x}$. For the sake of simplicity, we consider the case $k=1$, that is, the second order difference equation

$$
x_{n+1}=\alpha x_{n}+f\left(x_{n-1}\right), \quad n=0,1, \ldots,
$$

In this case, the criterion (1.6) for the local asymptotic stability reads $\left|f^{\prime}(\bar{x})\right|<1$. On the other hand, Proposition 1.1 gives the global asymptotic stability under condition $\left|f^{\prime}(\bar{x})\right| \leq$ $1-\alpha$, which is sharp when $\alpha$ is close to 0 . For $\alpha$ close to 1 , Corollary 3.6 gives better approximations. Indeed, it is easy to check that condition (3.4) always holds when $\bar{x}$ is locally asymptotically stable. Therefore, Corollary 3.6 ensures the convergence of all solutions of equation (3.9) to $\bar{x}$ when $\left|f^{\prime}(\bar{x})\right|>1-\alpha$ and $\max \left\{\left|f^{\prime}(x)\right|: x \in[a, b]\right\} \leq \alpha^{2}$, where $\{a, b\}$ is the 2-cycle of $(1-\alpha)^{-1} f(x)$. 
In the literature, there are several conjectures regarding the local and global asymptotic stability of the equilibrium for delay differential and difference equations. For the delay equation (1.2), it was suggested in [15], the equivalence between the local and global asymptotic stability conditions when $f$ satisfies $(\mathrm{H})$, which has as a limit case $(\delta=0)$ the celebrated Wright conjecture [13, p. 125].

For the discrete version of the Nicholson's blowflies equation, a similar conjecture was posed in ref. [6]. We guess that the following statement is true, although we are not able to prove it at this moment.

CONJECTURE 3.9 Assume that $(H)$ holds. If $\bar{x}$ is asymptotically stable then all solutions of equation (1.1) converge to $\bar{x}$.

In view of the above discussion for $k=1$, we propose the following open problem as the first step in proving the conjecture:

Open problem. Investigate if, for $k=1, \bar{x}$ attracts all solutions of equation (1.1) when (H) holds and $\left|f^{\prime}(\bar{x})\right|<1$.

\section{Applications}

In this section, we apply some of our previous results to state detailed global stability results for equations (1.3) and (1.4). We first consider the Mackey-Glass type equation (1.3). In this case, $f(x)=\beta /\left(1+x^{p}\right)$, and the equilibrium $\bar{x}$ is the unique positive solution of $x^{p+1}+x=\beta /(1-\alpha)$. From [11, Theorem 4.6.3], we know that $\bar{x}$ attracts all solutions of equation (1.3) if $p \leq 1$, so we can assume that $p>1$. One can check that $f^{\prime}(x)=-\beta p x^{p-1} /\left(1+x^{p}\right)^{2}<0$ and $(S f)(x)=\left(1-p^{2}\right) /\left(2 x^{2}\right)<0$ for all $x>0$; therefore, hypothesis (H) holds.

Next, Proposition 1.1 ensures that all solutions of equation (1.3) converge to $\bar{x}$ if $c=\left|h^{\prime}(\bar{x})\right| \leq 1$, that is, if

$$
\bar{x} \geq \frac{(p-1) \beta}{p(1-\alpha)}
$$

Now, setting $q(x)=x^{p+1}+x-\beta /(1-\alpha)$, we have that $q(0)<0$ and $\bar{x}$ is the unique positive root of $q$. Thus, equation (4.1) is equivalent to the inequality

$$
q\left(\frac{(p-1) \beta}{p(1-\alpha)}\right) \leq 0
$$

or, equivalently, $(\beta /(1-\alpha))^{p} \leq p^{p} /(p-1)^{p+1}$. This condition (with the strict inequality) was also obtained in [11, Theorem 4.6.3].

Taking into account the above discussion and using our results, we can state the following list of sufficient conditions for the convergence of all solutions to the equilibrium in equation (1.3), improving the corresponding estimations in refs. $[5,9,11,12]$. 
THEOREM 4.1 Each one of the following conditions is sufficient to ensure that all solutions of equation (1.3) converge to $\bar{x}$ :

(i) $p \leq 1$ (independent of $\alpha, \beta$ and $k$ );

(ii) $p>1$ and $(\beta /(1-\alpha))^{p} \leq p^{p} /(p-1)^{p+1}$ (independent of $k$ );

(iii) $p>1$ and

$$
\alpha^{k+1} \geq \frac{p^{2}-p}{p^{2}+1}
$$

(independent of $\beta$ );

(iv) $c>1$ and $\alpha^{k+1} \geq\left(c^{2}-c\right) /\left(1+c^{2}\right)$;

(v) $c>1$ and

$$
\alpha^{k+1} \geq \frac{\beta(k+1)^{2}}{2(3 k+4) p}(p+1)^{(p+1) / p}(p-1)^{(p-1) / p}
$$

(vi) $c>1, \alpha^{k} \geq\left(c^{2}-c\right) /\left(c^{2}+1\right)$, and

$$
\alpha^{k+1} \geq \frac{\beta}{4 p}(p+1)^{(p+1) / p}(p-1)^{(p-1) / p} ;
$$

(vii) $c>1$ and inequalities (3.8), (4.4) hold.

(viii) $c>1$ and $\min \left\{\left|f^{\prime}(a)\right|,\left|f^{\prime}(b)\right|\right\} \geq \alpha^{k+1} \geq(c-1) /(c+1)$, where $(a, b)$ is the unique 2-cycle of $h(x)=(1-\alpha)^{-1} f(x)=(1-\alpha)^{-1} \beta /\left(1+x^{p}\right)$.

Proof As it was mentioned above, (i) and (ii) follow, respectively from refs. [11] and [12]. To prove (iii), let us observe that $0<c=p-\beta^{-1} p(1-\alpha) \bar{x}<p$, and function $\gamma(x)=$ $\left(x^{2}-x\right) /\left(\lambda(x-1)+x^{2}+1\right)$ is increasing for $x>1$. Since equation (3.1) always holds for $\lambda=0$, Theorem 3.3 (1) ensures that $\bar{x}$ is a global attractor if $p>1$ and equation (4.2) holds. Analogously, condition (iv) follows directly from Theorem 3.3 (1), taking $\lambda=0$.

Next, it is easy to check that

$$
\max _{x \geq 0}\left\{\left|f^{\prime}(x)\right|\right\}=\frac{\beta}{4 p}(p+1)^{(p+1) / p}(p-1)^{(p-1) / p} .
$$

Hence, equation (4.3) implies equation (2.13), and then the result in (v) follows from Theorem 2.7. Next, from equation (4.5), we can see that equation (3.7) is true if equation (4.4) holds. Hence, (vi) and (vii) follow from Corollary 3.6 and 3.8, respectively. Finally, (viii) is a straightforward consequence of Corollary 3.5.

Remark 4.2 Of course, the remainder conditions in Theorem 3.3 can be applied to equation (1.3) as well. For example, condition (iv) in Theorem 4.1 can be improved to

$$
c>1 \text { and } \alpha^{k+1} \geq\left(c^{2}-c\right) /\left(\lambda(c-1)+1+c^{2}\right),
$$

with $\lambda \leq 1$ satisfying equation (3.1). In particular, using the relations $h(0)<a<b<h^{2}(0)$, we can choose $\lambda=\min \left\{1,\left|f^{\prime}(h(0))\right|,\left|f^{\prime}\left(h^{2}(0)\right)\right|\right\}$ with independence of $k$. The same comment applies to condition (iii). 
To complete our discussion, we present two numerical examples. First one shows that condition (viii) works in some cases in which (i)-(vii) do not apply.

Example 4.3 Consider equation (1.3) with $\alpha=0.7, \beta=0.77, p=2$ and $k=7$, that is,

$$
x_{n+1}=0.7 x_{n}+\frac{0.77}{1+x_{n-7}^{2}}, \quad n=0,1, \ldots
$$

The equilibrium $\bar{x}$ is the unique real solution of $x^{3}+x=7.7 / 3$, with an approximate value of $\bar{x}=1.1287 \ldots$ On the other hand, $c=\left|h^{\prime}(\bar{x})\right|=1.12049 \ldots>1$, and $\alpha^{k+1}=$ 0.05764801 . One can check that (i)-(vii) in Theorem 4.1 do not hold.

Now, since $\left|h^{\prime}(\bar{x})\right|>1$, there is a unique pair $(a, b)$ such that $h^{2}(a)=a, h^{2}(b)=b$, and $a<\bar{x}<b$. In this case, $a=0.479005 \ldots$ and $b=2.08766 \ldots$. Next,

$$
\min \left\{\left|f^{\prime}(a)\right|,\left|f^{\prime}(b)\right|\right\}=\left|f^{\prime}(b)\right|=0.111975 \ldots>\alpha^{k+1}>0.0568202 \ldots=\frac{c-1}{c+1} .
$$

Therefore, condition (viii) in Theorem 4.1 ensures that $\bar{x}$ is globally attracting.

Our next numerical example shows that in general condition (3.5) does not guarantee that $\bar{x}$ is a global attractor if equation (3.6) does not hold.

Example 4.4 Consider equation (1.3) with $\alpha=0.96, \beta=3, p=7$ and $k=6$, that is,

$$
x_{n+1}=0.96 x_{n}+\frac{3}{1+x_{n-6}^{7}}, \quad n=0,1, \ldots
$$

The equilibrium $\bar{x}$ takes an approximate value of $\bar{x}=1.71053 \ldots$ On the other hand, $c=\left|h^{\prime}(\bar{x})\right|=6.84035 \ldots>1, \quad$ and $\quad \alpha^{k+1}=0.751447>(c-1) /(c+1)=0.744909 \ldots$. Thus equation (3.5) holds. However, one can check that the characteristic polynomial associated to the linearized equation of (4.7) at $\bar{x}$ has a pair of conjugate roots with modulus greater than one. Hence, $\bar{x}$ is unstable. Of course, in this example (3.6) does not hold.

Finally, we discuss the application of our results to the Lasota-Wazewska type equation (1.4). In this case, $f(x)=p e^{-q x}$, and the equilibrium $\bar{x}$ is the unique positive solution of $p e^{-q x}=$ $(1-\alpha) x$. One can easily check that $(\mathrm{H})$ holds. Next, since $c=q \bar{x}$, condition $c \leq 1$ is equivalent to $p q \leq(1-\alpha) e$, which was already found in ref. [10]. If $c>1$, we can use Theorem 3.3 to obtain new conditions for the global attractivity of $\bar{x}$. Let us observe that $f^{\prime}$ is increasing and negative. Therefore, equation (3.1) holds if and only if $\alpha^{k+1} \leq \lambda^{-1}\left|f^{\prime}(b)\right|=\lambda^{-1} p q e^{-q b}$, while equation (2.8) holds if and only if $\alpha^{k+1} \geq \lambda^{-1}\left|f^{\prime}(a)\right|=\lambda^{-1} p q e^{-q a}$. Moreover, for $\lambda>1$,

$$
(S Z)(x)=\frac{\left(1-\alpha^{k}\right) p q^{3} e^{-q x}}{2(\eta(x))^{2}(1-\alpha)}\left(2(\lambda-1) \alpha^{k}-\frac{\left(1-\alpha^{k}\right) p q e^{-q x}}{(1-\alpha)}\right),
$$

where $\eta(x)=\alpha^{k}(\lambda-1)+\left(1-\alpha^{k}\right)(1-\alpha)^{-1} p q e^{-q x}$. Thus, $(S Z)(x) \leq 0$ in $[a, b]$ when $\lambda \leq$ $\lambda_{2}:=1+\left(1-\alpha^{k}\right) /\left(2 \alpha^{k}\right) a q$. Now, an application of Theorem 3.3 gives the following theorem, which improves the results in refs. [2], [5-7], [9-11]. 
THEOREM 4.5 Each one of the following conditions is sufficient to ensure that all solutions of equation (1.4) converge to $\bar{x}$ :

(i) $p q \leq(1-\alpha) e$ (independent of $k$ );

(ii) $p q>(1-\alpha)$ e and there exists $\lambda \in(0,1]$ such that

$$
\frac{p q e^{-q b}}{\lambda} \geq \alpha^{k+1} \geq \frac{c^{2}-c}{\lambda(c-1)+1+c^{2}},
$$

where $b$ is the unique solution in $(\bar{x}, \infty)$ of $h^{2}(x)=x$, with $h(x)=(1-\alpha)^{-1} p e^{-q x}$.

(iii) $p q>(1-\alpha)$ and there exists $1 \leq \lambda \leq \lambda_{2}$ such that equation (3.3) holds and $\alpha^{k+1} \geq$ $\lambda^{-1} p q e^{-q a}$, where a is the unique solution in $(0, \bar{x})$ of $h^{2}(x)=x$.

Notice that for $\lambda \leq p q e^{-q b}$ the first inequality in equation (4.8) trivially holds and hence Theorem 4.5 improves the result for equation (1.4) obtained by applying Theorem 1.2.

Theorem 2.7 and Corollary 3.8 provide the following conditions easier to verify:

COROLlaRy 4.6 Assume that $p q>(1-\alpha)$ e. Then all solutions of equation (1.4) converge to $\bar{x}$ if either $\alpha^{k+1} \geq 2 p q e^{-q a}(k+1)^{2} /(3 k+4)$, or $\alpha^{k+1} \geq p q e^{-q a}$ and condition (3.8) holds.

One can also use the rest of Theorem 3.3 to find other global attractivity criteria. For example, for the Lasota-Wazewska type equation (1.4), the function $F_{1}(x)$ has a critical point $x_{1}^{*}$ given by

$$
x_{1}^{*}=\frac{1}{q} \ln \left(\frac{p q}{\alpha^{k+1}}\right) .
$$

Therefore, the inequality

$$
F_{1}\left(x_{1}^{*}\right)+\left(\alpha-\alpha^{k+1}\right) a=\alpha^{k+1} x_{1}^{*}+\frac{\alpha^{k+1}}{q}+\left(\alpha-\alpha^{k+1}\right) a \geq x_{1}^{*}
$$

is equivalent to

$$
\ln \left(\frac{p q}{\alpha^{k+1}}\right) \leq \frac{\alpha^{k+1}}{1-\alpha^{k+1}}+\frac{\alpha-\alpha^{k+1}}{1-\alpha^{k+1}} a q .
$$

Hence, equation (2.12) holds if and only if equation (4.9) is satisfied. Now, applying Theorem 3.3(4), we see that all solutions of equation (1.4) converge to $\bar{x}$ provided that conditions (3.4) and (4.9) are satisfied.

\section{Acknowledgements}

E. Liz was supported in part by M.C.T. (Spain) and FEDER, under project BFM2001-3884C02-02.

\section{References}

[1] Cooke, K.L. and Ivanov, A.F., 2000, On the discretization of a delay differential equation, Journal of Difference Equations and Applications, 6, 105-119. 
[2] El-Morshedy, H.A., 2003, The global attractivity of difference equations of nonincreasing nonlinearities with applications, Computers and Mathematics with Applications, 45, 749-758.

[3] Erbe, L.H., Xia, H. and Yu, J.S., 1995, Global stability of a linear nonautonomous delay difference equation, Journal of Difference Equations and Applications, 1, 151-161.

[4] Gopalsamy, K., Trofimchuk, S.I. and Bantsur, N.R., 1998, A note on global attractivity in models of haematopoiesis, Ukraine Mathematical Journal, 50, 5-12.

[5] Graef, J.R. and Qian, C., 1999, Global stability in a nonlinear difference equation, Journal of Difference Equations and Applications, 5, 251-270.

[6] Györi, I. and Trofimchuk, S., 2000, Global attractivity and persistence in a discrete population model, Journal of Difference Equations and Applications, 6, 647-665.

[7] Hamaya, Y., 1997, Global attractivity in Wazewska-Lasota difference model, Differential Equations and Dynamic Systems, 5, 187-195.

[8] an der Heiden, U. and Mackey, M.C., 1982, The dynamics of production and destruction: analytic insight into complex behavior, Journal of Mathematical Biology, 16, 75-101.

[9] Ivanov, A.F., 1994, On global stability in a nonlinear discrete model, Nonlinear Analysis, 23, $1383-1389$.

[10] Karakostas, G., Philos, Ch.G. and Sficas, Y.G., 1991, The dynamics of some discrete population models, Nonlinear Analysis, 17, 1069-1084.

[11] Kocić, V.L. and Ladas, G., 1993, Global Behavior of Nonlinear Difference Equations of Higher Order with Applications, Mathematics and its Applications (Dordrecht: Kluwer Academic Publishers Group), Vol. 256.

[12] Krause, U. and Pituk, M., 2004, Boundedness and stability for higher order difference equations, Journal of Difference Equations and Applications, 10, 343-356.

[13] Kuang, Y., 1993, Delay Differential Equations with Applications in Population Dynamics (Boston, MA: Academic Press).

[14] Kuruklis, S.A., 1994, The asymptotic stability of $x_{n+1}-a x_{n}+b x_{n-k}=0$, Journal of Mathematical Analysis and Applications, 188, 719-731.

[15] Liz, E., Tkachenko, V. and Trofimchuk, S., 2003, A global stability criterion for scalar functional differential equations, SIAM Journal on Mathematical Analysis, 35, 596-622.

[16] Singer, D., 1978, Stable orbits and bifurcation of maps of the interval, SIAM Journal on Applied Mathematics, 35, 260-267. 